\title{
Fecal Microbial Enterotypes Differentially Respond to a High-fat Diet Based on Sex in Fischer-344 Rats
}

\author{
Soo In Choi', Nayoung Kim,2, Ryoung Hee Nam1, Ji Hyun Park², Heewon Nho', Jeong Eun Yu', Chin-Hee Song', \\ Sun Min Lee', Dong Ho Lee ${ }^{1,2}$ \\ ${ }^{1}$ Department of Internal Medicine, Seoul National University Bundang Hospital, Seongnam, ${ }^{2}$ Department of Internal \\ Medicine and Liver Research Institute, Seoul National University College of Medicine, Seoul, Korea
}

The gut microbiota interacts with the host gut environment, which is influenced by such factors as sex, age, and host diet. These factors induce changes in the microbial composition. The aim of this study was to identify differences in the gut microbiome of Fisher-344 (F344) rats fed a high-fat diet (HFD), depending on their age and sex. Fecal microbiomes from 6-, 31-, and 74-week-old, and 2-year-old both male and female rats (corresponding to 5-, 30-, 60-, and 80-year-old humans) were analyzed using 16S rRNA gene sequencing, phylogenetic investigation of communities by reconstruction of unobserved states, and enterotype (E) assessment. Moreover, the effect of an HFD on colonic epithelial cells was measured using real-time quantitative PCR. Alpha diversity decreased in the HFD group regardless of age and sex. Based on the enterotype clustering of the whole fecal microbiome, clusters from male rats were divided into E1 and E2 enterotypes, while clusters from female rats were divided into E1, E2, and E3 enterotypes. The female E3 group showed a significantly high abundance in the Ruminococcus genus and expression of T/r2 mRNA, which may reflect compensation to the HFD. Moreover, the female E3 group showed a lower ratio of opportunistic pathogenic strains to commensal strains compared to the female E2 group. Administration of an HFD influenced the rat fecal microbiota in all assessed age groups, which could be further differentiated by sex. In particular, female rats showed a compensatory enterotype response to an HFD compared to male rats.

Key Words Rats, Diet, high-fat, Microbiota, Aging, Sex

\section{INTRODUCTION}

The gut microbiota inhabits the gastrointestinal tract and interacts with the host gut environment. Various microorganisms colonize the gastrointestinal tract starting at birth, and their composition changes depending on the health status of the host $[1,2]$. In addition, gut microbiota ferments undigested food that man ingests and produces metabolites that can affect host health $[3,4]$. A high-fat diet (HFD) is associated with obesity and metabolic syndromes, which are major risk factors for cardiovascular disease and various types of cancer, such as prostate cancer [5]. Furthermore, the consumption of an HFD results in chronic systemic inflammation in the liver, adipose tissue, skeletal muscle, and intestine [6]. Also, dietary patterns affect the microbial diversity and the community structure in the gut microbiota $[7,8]$. Interestingly, a change in the host's diet, even for a short period of time, may alter the microbiota, which persists for a few days. Indeed, short-term dietary changes even within 2 days alter the gut microbiota [3].

Apart from diet, aging also induces changes in the gut microbiome. Aging promotes physiological, genetic, immunological, and metabolic changes to occur throughout the body, thereby affecting the gut microbiota [9]. One of the examples of changes in the gut microbial composition is the Clostridium difficile infection, in which the rate of incidence is increased in elders $[10,11]$. As the host ages, the gut microbiota also slowly undergoes transitions from that of a younger person to that of an older person.

Recently, it has been shown that various health conditions are influenced by sex [12]. Similarly, sex differences in the gut microbiome have also been reported [13]. Analysis of the fecal bacterial community, comparing a rodent chow diet and oligofructose supplemented diet to rats, demonstrated that

Received December 13, 2021, Revised December 23, 2021, Accepted December 24, 2021 
sex differences in microbial metabolism affected inflammatory cytokine expression. Thus, increases in anti-inflammatory cytokine expression (e.g., interleukin [IL]-10) in females [14] and proinflammatory cytokine expression (e.g., IL-6) in males were observed [15]. Another study also reported that sex and hormonal changes affect the gut microbiome diversity and composition in 89 different inbred strains of mice [16].

The production of various metabolites from gut microorganisms, such as short-chain fatty acids (SCFAs like acetate, propionate, and butyrate) is one of the ways that the gut microbiome affects host health [3]. Among SCFAs, microbiome-derived butyrate has been reported to promote epithelial barrier function through the IL-10 receptor [17].

Previously, we reported the effect of an HFD on a young (6-week-old) and older (2-year-old) Fischer-344 (F344) rat gut microbiome, in both male and female rats [18]. The results suggested that sex influences the response of gut microbiota to an HFD, particularly in old age. We have also reported that the butyrate level in the cecum was significantly increased in 31-week-old rats among a cohort of 6-, 31-, and 74-week-old, and 2-year-old male F344 rats [19]. With this background, we hypothesized that 31- and 74-week-old rats might show a different microbiota pattern compared to 6-week-old and 2-yearold rats, depending on their sex, upon exposure to an HFD for 8 weeks. Therefore, the aim of this study was to identify differences in the gut microbiome of 6-, 31-, and 74-week-old and 2-year-old F344 rats fed an HFD based on the sex of the animals.

\section{MATERIALS AND METHODS}

\section{Animals and diets}

F344 rats, aged 6-, 31-, and 74-week, and 2-year-old were used in this study (OrientBio, Seongnam, Korea) (Table S1) $[20,21]$. Animals were housed in a specific-pathogen-free room at $23^{\circ} \mathrm{C}$ with a $12 / 12 \mathrm{~h}$ light/dark cycle and given unlimited access to food and water. The rats were divided into groups that were fed ad libitum, with two different commercial diets: a normal diet and an HFD (normal chow: $3.85 \mathrm{kcal} /$ g; HFD: $5.24 \mathrm{kcal} / \mathrm{g}, 60 \% \mathrm{kcal}$ derived from fat) (Product \#D12492; Research Diets, Inc., New Brunswick, NJ, USA). Fecal samples were collected after 8-week administration of normal diet or HFD and stored at $-80^{\circ} \mathrm{C}$. The study was performed following the procedures in the Guide for the Care and Use of Laboratory Animals in Korea. All protocols used were approved by the Institutional Animal Care and Use Committee (IACUC) of Seoul National University Bundang Hospital (Permission No. BA1506-178/027-01).

\section{Fecal sample collection and metagenome sequencing}

Bacterial genomic DNA was extracted from the frozen fecal samples using a QIAamp DNA Stool Mini Kit (Qiagen, Hilden, Germany) according to the manufacturer's recommendations.
DNA quantity and quality were measured using a NanoDrop 1000 spectrophotometer (Thermo Fisher Scientific, Waltham, MA, USA) and electrophoresed using $2 \%$ agarose gel. For the preparation of MiSeq library amplicons, the target gene, 16S rRNA V3-V4 region, was first amplified using the 341-F (5'-TCG TCG GCA GCG TCA GAT GTG TAT AAG AGA CAG CCT ACG GGN GGC WGC AG-3') and 805-R (5'-GTC TCG TGG GCT CGG AGA TGT GTA TAA GAG ACA GGA CTA CHV GGG TAT CTA ATC C-3') primers. Secondly, the V3V4 PCR amplicons were linked to the Illumina indices and adapters from Nextera ${ }^{\circledR}$ XT Index Kit (Illumina, San Diego, CA, USA). Short DNA fragments were eliminated using the FavorPrep $^{\mathrm{TM}}$ DNA purification kit (Favorgen, Pingtung, Taiwan). To quantify the PCR amplicons, we used the Quant-iT ${ }^{\mathrm{TM}}$ PicoGreen $^{\text {TM }}$ dsDNA Assay Kit (Thermo Fisher Scientific). Sequencing was performed at Chunlab Inc. (Seoul, Korea) using the MiSeq system (Illumina).

Non-specific, non-target, and chimeric amplicons were removed in the quality control process to exclude reads with short lengths and low Q-values from the pre-filter. Using operational taxonomic unit (OTU) information (number of OTUs and sequences in each OTU), alpha diversity indices, such as abundance-based coverage estimator (ACE), Chao1, Jackknife, Shannon indices, Simpson indices, phylogenetic diversity, and Good's library coverage) were analyzed using EzBioCloud (Chunlab Inc.) (Table S2). To visualize sample differences, principal coordinates analysis was performed with unweighted UniFrac. Averaged taxonomic compositions (at phylum and family level) bar charts and selected taxa were created using GraphPad Prism (ver. 8.01; GraphPad Software, Inc., San Diego, CA, USA).

To predict the functional capabilities of the microbial community, we performed a phylogenetic investigation of communities by reconstruction of unobserved states.

\section{Enterotype clustering and LEfSe analysis}

The enterotype classification of the gut microbiota at the genus level was calculated using the R package "clusterSim" [22]. The optimal cluster number was determined by maximizing the value of the Calinski-Harabasz index [23].

Based on the relative taxonomic abundances, the taxonomic biomarker discovery and related statistical significance were assessed by the linear discriminant analysis effect size (LEfSe) method [24]. The criteria for conducting the LEfSe analysis were as follows: 1) an alpha value for the factorial Kruskal-Wallis $\mathrm{H}$ test between assigned taxa compared to that of the groups with less than $0.05,2$ ) an alpha value for the pairwise Wilcoxon test among the taxonomic compositions of less than $0.05,3$ ) a threshold of the logarithmic linear discriminant analysis (LDA) score for discriminative features less than 2.0, and 4) a multi-class analysis set as all-against-all. After this process, we simplified the LEfSe plot by 5 ) overlapped bacteria selection, 6) searched and classified the bacterial characteristics regarding reported papers: commensal strains 
A Verrucomicrobia

Firmicutes $\square$ Bacteroidetes $\square$ Proteobacteria

Etc. (under $1 \%$ in average)

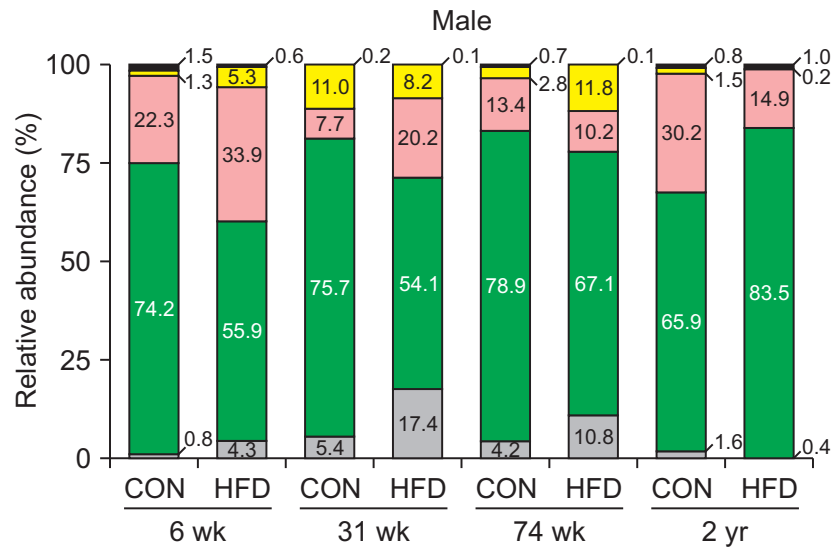
Female
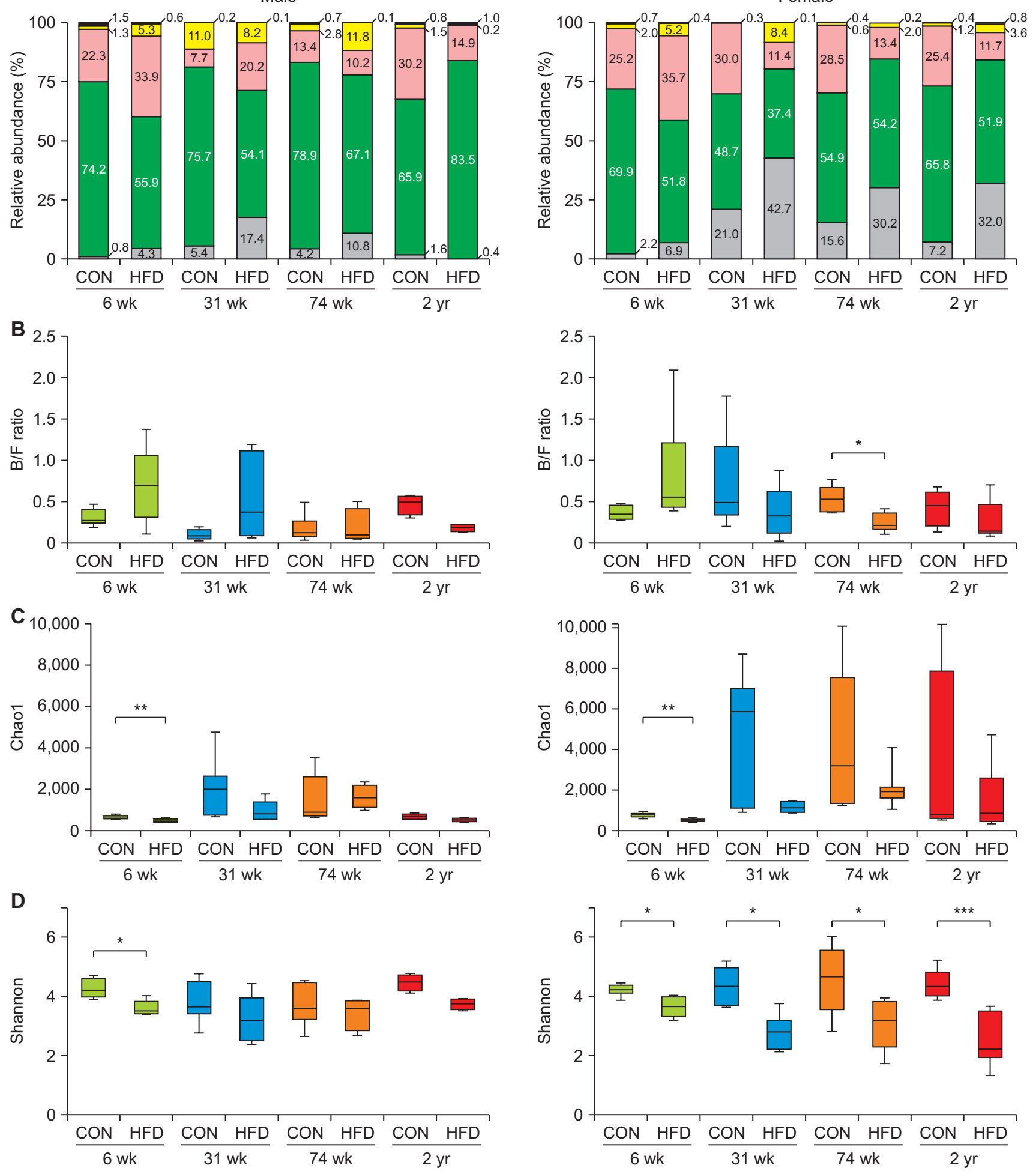

Figure 1. Comparison of age-associated variations in the fecal microbiomes in the male and female rats fed the control diet (CON) or highfat diet (HFD). Fecal samples were collected and subjected to measurement of phylum composition (A), the Bacteroides to Firmicutes (B/F) ratio (B), Chao1 indicating the species richness (C), and Shannon indicating the diversity of species (D). Data are expressed as the median, Q1 (25\%) and Q3 $(75 \%)$. Whiskers show the minimum and maximum values. The statistical significance was determined by the Mann-Whitney test. CON, control diet; HFD, high-fat diet. ${ }^{*} P<0.05,{ }^{* \star} P<0.01$, and ${ }^{* * *} P<0.001$. 
and opportunistic pathogenic strains at the genus level.

\section{Real-time quantitative PCR (RT-qPCR)}

Total RNA was extracted from the descending colon tissue using TRIzol ${ }^{\circledR}$ reagent (Invitrogen, Carlsbad, CA, USA) as recommended by the manufacturer, and the collected RNA was purified using an RNeasy Mini kit (Qiagen). Complementary DNA synthesis was performed using $1 \mu \mathrm{g}$ of total RNA with the High-Capacity cDNA kit (Applied Biosystems, Foster City, CA), according to the manufacturer's instructions. RT-qPCR was performed in triplicate using a StepOnePlus ${ }^{\mathrm{TM}}$ Real-time PCR (Applied Biosystems, Waltham, MA, USA) with SYBR ${ }^{\circledR}$ Premix Ex Taq ${ }^{\mathrm{TM}}$ (TaKaRa Bio; Shiga, Japan) according to the manufacturer's instructions with designed primer (Table S3). The expression level of mRNA for the target genes was compared with endogenous control $\beta$-actin using the $2-\Delta \Delta \mathrm{Ct}$ method.

\section{Statistical analysis}

Statistical calculations, except for those on the pyrosequencing data, were performed using PASW Statistics version 18.0.0 (SPSS Inc., Released 2009; PASW Statistics for Windows, Version 18.0.; SPSS Inc., Chicago, IL, USA). The groups were compared using the Mann-Whitney U-test (also known as the Wilcoxon rank-sum test). A $P$-value $<0.05$ was considered to be statistically significant.

\section{RESULTS}

\section{Compositional changes depend on sex, age, and diet}

The daily calorie intake and the gained body weight during the 8-week HFD feeding period were reported in our previous study [25]. The weight gain was decreased with age both in male and female rats. When we compared taxonomic compositional differences between the HFD-fed group and the normal diet group at the phylum level (Fig. 1), Bacteroidetes decreased in the HFD-fed group in male (2-year-old, $P=$ $0.021)$ and female rats (31-week-old, $P=0.016$; 74-week-old, $P=0.015$; 2-year-old, $P=0.010)$. In the case of Firmicutes, the 6-week-old male $(P=0.037)$ and female $(P=0.006)$ HFD-fed groups showed decreased relative abundance compared to the normal diet group. Verrucomicrobia increased in 6-week- $(P=0.004)$ and 31-week-old $(P=0.031)$ male rats and 6-week-old $(P=0.016)$ and 2-year $(P=0.008)$ female rats (Fig. 1A). In terms of the Bacteroidetes to Firmicutes $(B /$ F) ratio, the 74-week-old female group showed a decreased ratio in the HFD-fed group compared to the normal diet group (Fig. 1B). In contrast, the Chao1 index, which is an alpha-diversity index indicating species richness, was decreased in the 6-week-old male $(P=0.004)$ and female $(P=0.006)$ HFD-fed groups compared to the normal diet-fed group (Fig. $1 \mathrm{C})$. Moreover, the Shannon diversity index, an indicator of diversity in species, decreased in all female HFD-fed groups (6-week-old, $P=0.010$; 31-week-old, $P=0.010$; 74-week-old, $P=0.020 ; 2$-year-old, $P<0.001)$ compared to the normal diet-fed group (Fig. 1D).

\section{Taxonomic difference between the HFD-fed group and normal diet group}

At the genus level, the Pseudoflavonifractor showed a decreasing tendency in all the HFD-fed groups and significantly decreased in the 74-week-old $(P=0.002)$ and 2-year-old $(P=0.041)$ female HFD-fed groups compared to the normal diet group (Fig. 2A). Akkermansia muciniphila species showed a significant increase in the 6-week-old $(P=0.004)$ and 31-week-old $(P=0.031)$ male groups and 6-week-old $(P=0.016)$ and 2-year-old $(P=0.008)$ female groups (Fig. 2B). Phascolarctobacterium faecium species significantly increased in the HFD-fed groups of 6-week-old $(P=0.002)$, 31-week-old $(P=0.001)$, and 74-week-old $(P=0.029)$ males, and 6-week-old $(P=0.022)$ and 31-week-old $(P=0.002)$ females compared to the normal diet group (Fig. 2C). Contrarily, the number of unknown species PAC001125 s was significantly increased in the 2-year-old male HFD-fed group ( $P=$ 0.021 ) (Fig. 2D). The result of LEfSe analysis also showed a similar tendency (Figure S1). Except for the 2-year-old male group, Phascolarctobacterium faecium species increased in HFD fed male group, and Akkermansia muciniphila species increased in HFD fed groups of 6-week and 2-year-old female and 31-week-old male rats. However, it was hard to find any pattern differences between normal diet and HFD fed groups except Phascolarctobacterium faecium and Akkermansia muciniphila species.

\section{Enterotypes of microbiomes}

The principal coordinates analysis plot (Figure S2) was separated by diet while sex-and age-related separation did not appear. Therefore, we performed enterotype clustering for each sex using the Calinski-Harabasz index (Figure S3). As the Calinski-Harabasz index was maximized at two with males and three with females, enterotypes were separated into Enterotype1 (E1) and Enterotype2 (E2) in males (Figure S3; Fig. 3A), while females were separated into Enterotype1 (E1), Enterotype2 (E2), and Enterotype3 (E3) (Figure S3; Fig. $3 \mathrm{~B})$. Male $\mathrm{E} 1$ was composed of all normal diet groups and three of the 2-year-old HFD-fed group. The rest of the rats in the HFD-fed group were included in E2 (Table 1). Female E1 was composed of four of the 6-week-old, six of the 31-weekold, four of the 74-week-old, and five of the 2-year-old normal diet groups. Female E2 was composed of one of the 6-weekold normal diet group, six of the 6-week-old, one of the 31-week-old, five of the 74-week-old, and five of the 2-yearold HFD-fed groups. Female E3 was composed of one of the 6 -week-old, one of the 74-week-old, and one of the 2-yearold normal diet groups, as well as five of the 31-week-old, six of the 74-week-old, and five of the 2-year-old HFD-fed groups (Table 1). 
A

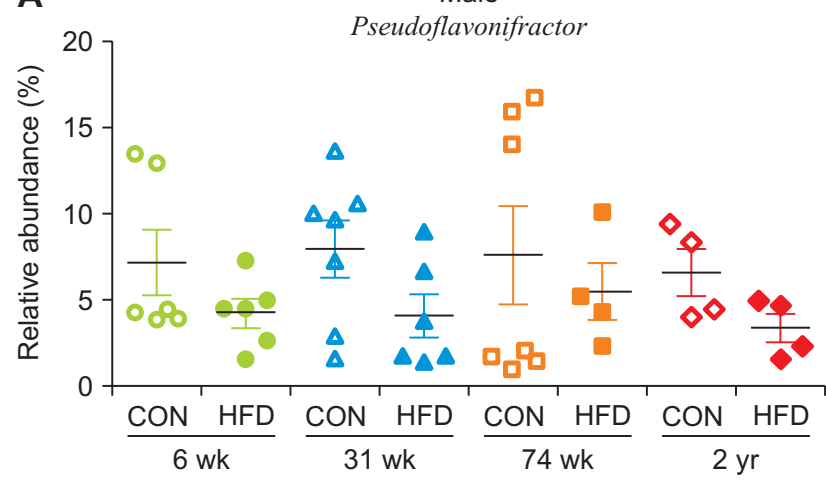

B

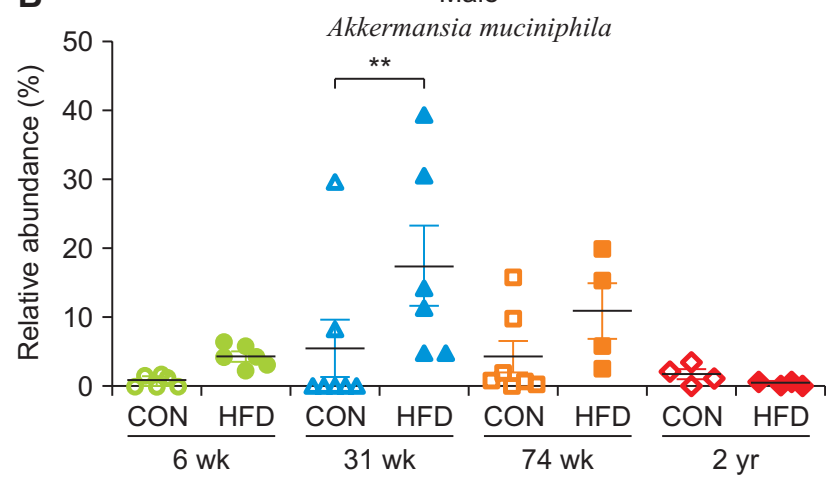

C

Phascolarctobacterium faecium

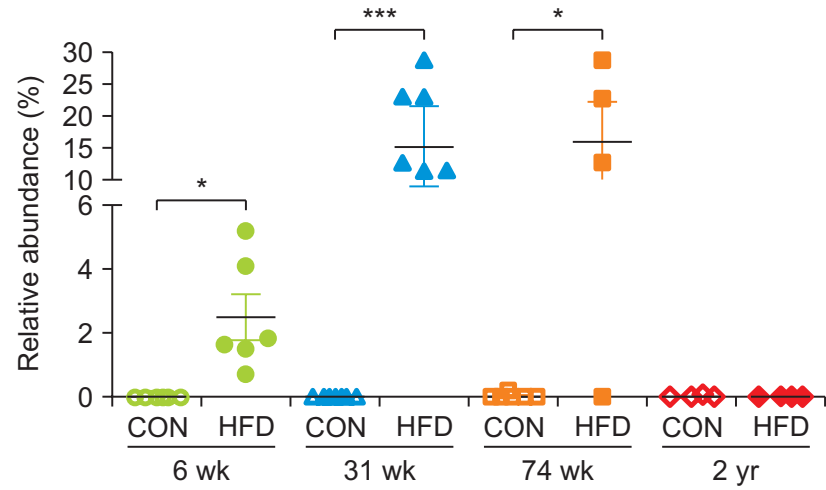

D

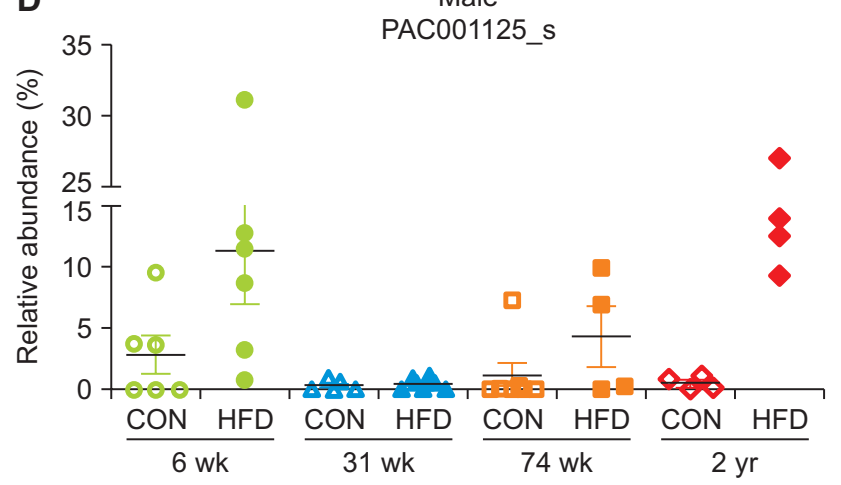

Female

Pseudoflavonifractor

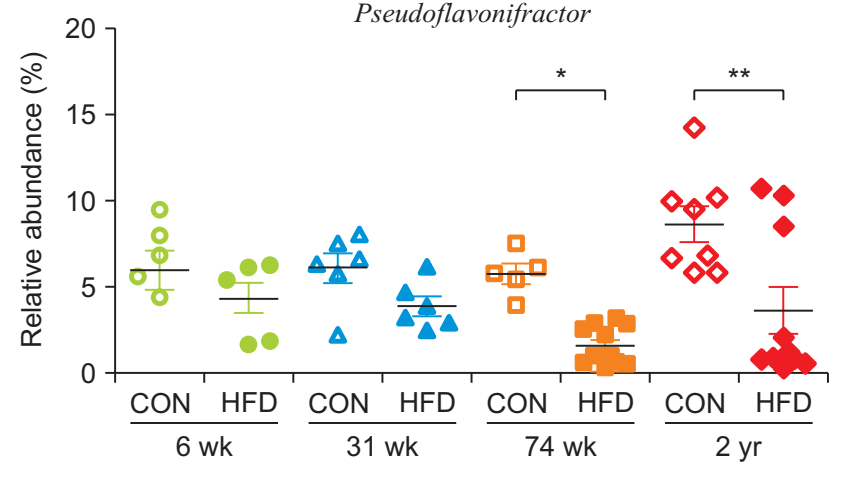

Female

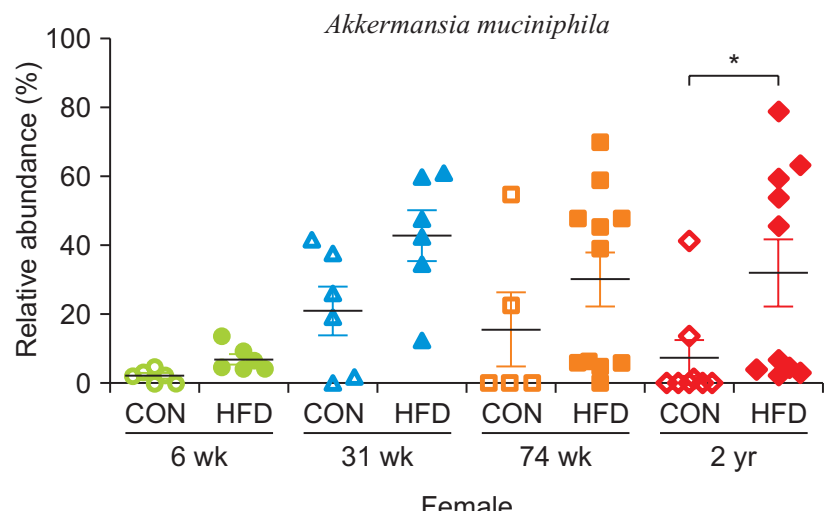

Phascolarctobacterium faecium

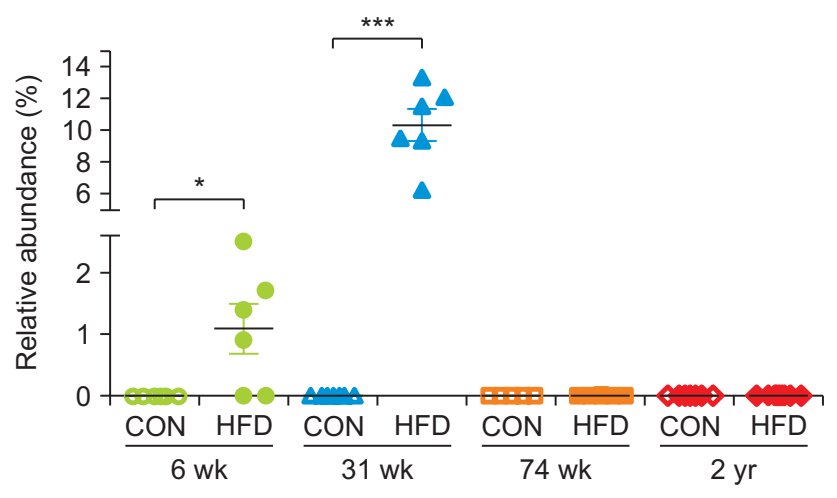

Female

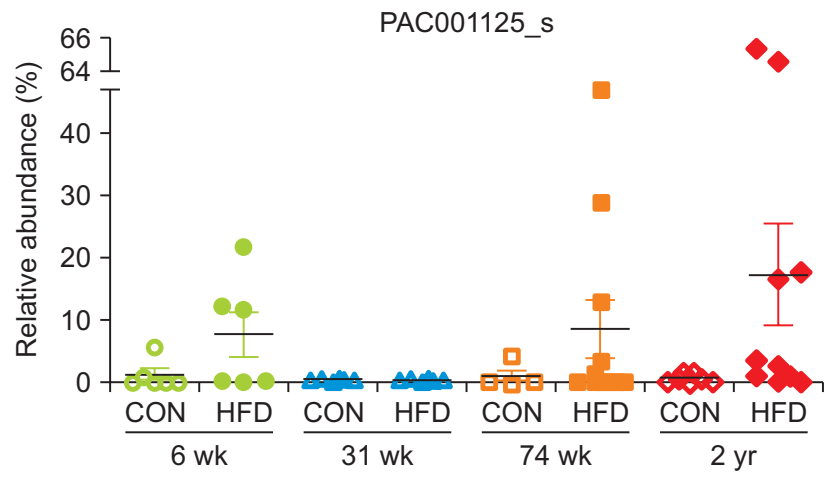

Figure 2. Alterations in the abundance ratio of fecal microbial species depending on age and diet. The relative abundance of Pseudoflavonifractor (A), Akkermansia muciniphila (B), Phascolarctobacterium faecium (C), and PAC001125_s (D). Collection of fecal samples and their microbiome analysis was conducted as described in "MATERIALS AND METHODS". The statistical significance was determined by the MannWhitney test; ${ }^{*} P<0.05,{ }^{* *} P<0.01$, and ${ }^{* *} P<0.001$. 

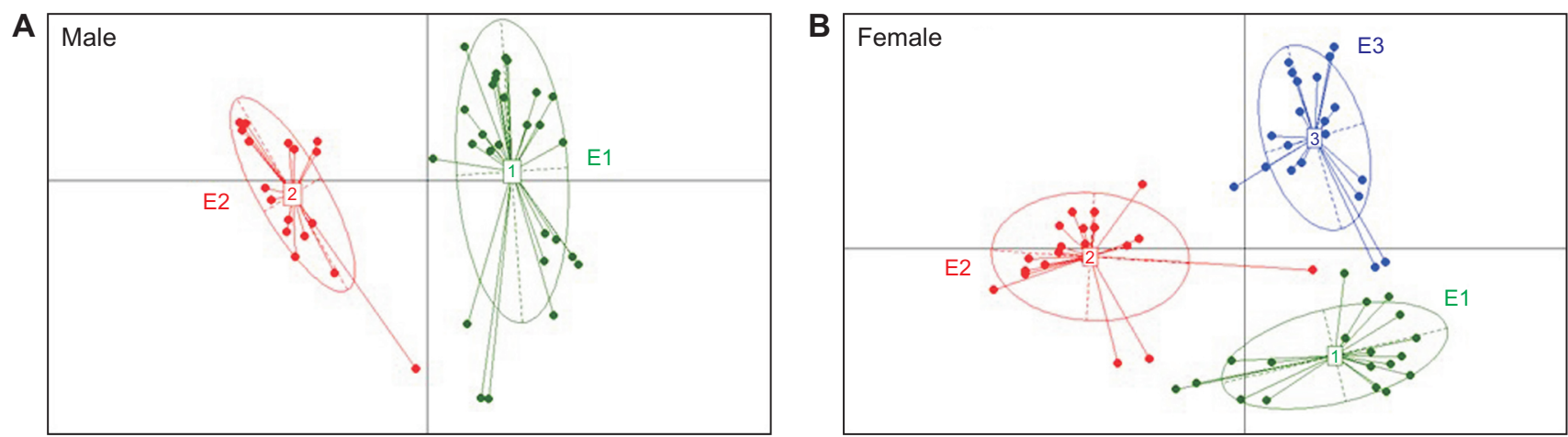

Figure 3. Enterotype-like clustering analysis in the fecal samples of male and female rats fed control (CON) or high-fat diet (HFD). There are two fecal enterotypes (E1 and E2) in males (A) and three enterotypes (E1, E2, E3) in female rats (B). The optimal cluster number was determined by maximizing the Calinski-Harabasz index value (Figure S2).

Table 1. The predominant species in each enterotype of male and female rat samples shown in Figure 3

\begin{tabular}{clccccc}
\hline Enterotype & Diet & 6 wk & 31 wk & 74 wk & 2 yr & Dominant bacteria species \\
\hline Male & & & & & & \\
E1 & CON & 6 & 7 & 7 & 4 & PAC001490_s (4.0\%), EU622773_s (3.7\%), PAC002478_s (3.2\%) \\
& HF & 0 & 0 & 0 & 3 & \\
E2 & CON & 0 & 0 & 0 & 0 & $\begin{array}{c}\text { Phascolarctobacterium faecium (11.2\%), Akkermansia muciniphila (10.2\%), } \\
\text { Female }\end{array}$ \\
E1 & HF & 6 & 6 & 4 & 1 & Bacteroides sartorii (9.8\%) \\
& CON & 4 & 6 & 4 & 5 & Akkermansia muciniphila (10.8\%), EU622773_s (6.7\%), Bacteroides sartorii (3.5\%) \\
E2 & HF & 0 & 0 & 0 & 0 & \\
& CON & 1 & 0 & 0 & 2 & Akkermansia muciniphila (53.1\%), JAGT_s group (6.4\%), Bacteroides sartorii (4.3\%) \\
E3 & HF & 6 & 1 & 5 & 5 & \\
& CON & 1 & 0 & 1 & 1 & PAC001125_s (16.2\%), Bacteroides sartorii (9.6\%), PAC002478_s (5.1\%)
\end{tabular}

Values are presented as number of rats. E, enterotype; CON, control diet; HF, high-fat.

\section{Compositional feature of the enterotypes}

We plotted the taxonomic composition of each enterotype, and observed clear differences at the phylum and genus levels (Fig. 4). In the case of males, E2 showed an increase in population of Verrucomicrobia $(P<0.001)$, Proteobacteria $(P=0.002)$, and Bacteroidetes $(P=0.197)$ phyla, while Firmicutes phyla decreased significantly $(P=0.004)$ compared to the composition of E1 (Fig. 4A). Furthermore, Bacteroides genus were increased significantly in E2 and Prevotella genus increased significantly in $E 1$, both of which belong to Bacteroidetes phyla (Fig. 4B). In enterotypes from females, Proteobacteria phyla increased significantly in E2 (E1 vs. E2, $P<0.001$; E2 vs. E3, $P=0.001)$, whereas E3 showed a significant decrease in Firmicutes phyla (E1 vs. E3, $P<0.001$; E2 vs. E3, $P<0.001$ ) and Bacteroidetes phyla (E1 vs. E3, $P<0.001$; E2 vs. E3, $P=0.006$ ), and a significant increase in Verrucomicrobia phyla (E1 vs. E3, $P<0.001$; E2 vs. E3, $P<0.001$ ) (Fig. 4C). Furthermore, Bacteroides genus was increased significantly in E2 (E1 vs. E2, $P=0.002$; E2 vs. E3, $P=0.001)$, Prevotella genus increased significantly in E1 (E1 vs. E2, $P<0.001$; E1 vs. E3, $P<0.001)$, and Ruminococcus increased significantly in E3 (E1 vs. E3, $P=0.005$; E2 vs. E3,
$P=0.008)($ Fig. 4D)

\section{Characteristics of the enterotypes}

The results from the LEfSe analysis (Fig. 5) indicated that the microbiomes of male E1 and female E1 were dominated by Monoglobus pectinilyticus and Acutalibacter muris species compared to male E2 or female E2 and female E3 (Fig. 5A-5C). In contrast, the microbiome of female E2 was dominated by Clostridium leptum, Desulfovibrio fairfieldensis, Bacteroides thetaiotaomicron, and Romboutsia timonensis species compared to female E1 and E3, which are known as opportunistic pathogenic strains (Fig. 5B and 5D). In female $\mathrm{E} 3$, Akkermansia muciniphila was the dominant species compared to female E1 and E2, which are known as commensal strains (Fig. 5C and 5D). From the difference in abundance between each enterotype, based on the LEfSe analysis, commensal strains and opportunistic pathogenic strains were categorized in accordance with previous studies, and their ratios were compared (Fig. 5E). Male E1 and female E1 dominated strains were all commensal bacteria. Meanwhile, female E2 had higher opportunistic pathogenic strains compared to female E3. Upon assessment of mRNA expression 


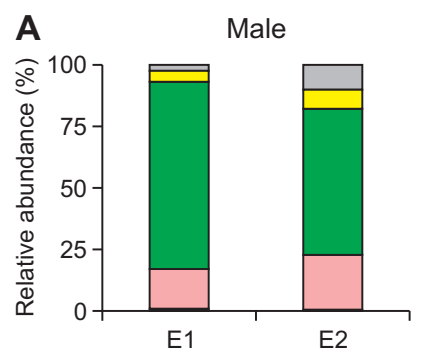

E1

Etc. (under $1 \%$ in average) Bacteroidetes

Firmicutes

Proteobacteria

$\square$ Verrucomicrobia

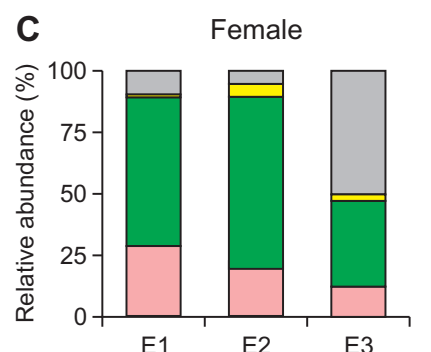

Etc. (under $1 \%$ in average)

Bacteroidetes

Firmicutes

Proteobacteria

Verrucomicrobia
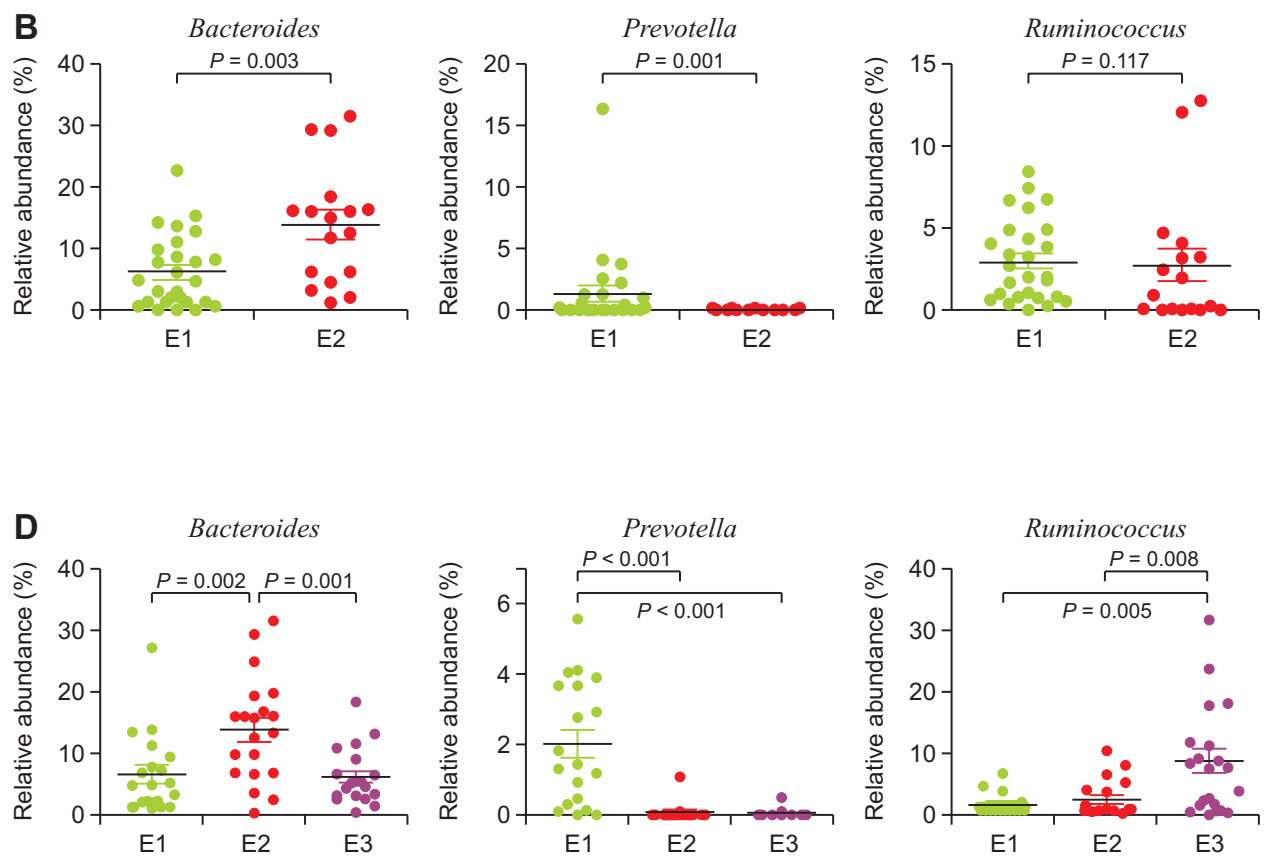

Figure 4. Comparisons of relative abundance of representative fecal microbial enterotypes. Fecal samples from the male (A, $B$ ) and female $(C, D)$ rats from control or high-fat diet-fed were collected and subjected to analysis of microbial enterotypes at the phylum level (A, C) and 3 representative human enterotypes (Bacteroides, Prevotella, and Ruminococcus) genera. The statistical significance was calculated by the MannWhitney test.

levels using RT-qPCR, male E1 was found to be significantly higher for Mucin 2 (Muc2) $(P<0.001)$ and Occludin $(O c l n)(P$ $=0.034$ ) compared to male E2 (Fig. 6A). In addition, female E1 was significantly higher for Muc2 (E1 vs. E2, $P=0.002$; E1 vs. E3, $P=0.001)$ and Ocln (E1 vs. E2, $P=0.018$; E1 vs. E3, $P=0.022)$ compared to female E2 and E3. Moreover, female E3 was significantly higher for Toll-like receptor 2 (TIr2) compared to female E2 $(P=0.010)$ (Fig. 6B).

\section{DISCUSSION}

In our present study, it was found that an HFD influenced fecal microbiota at all ages. Interestingly, the microbiota differed by sex, in a way that female rats showed a compensatory enterotype response to an HFD. In addition, an HFD lowered alpha diversity regardless of age and sex, suggesting that longterm administration of an HFD leads to intestinal microbial homeostasis. In agreement with a previous report [26], we observed an increase in the ratio of opportunistic pathogenic to commensal strains in the HFD groups, suggesting that an HFD not only destroys the diversity but also increases the chances of colonizing opportunistic strains.

The human gut microbiota mostly consists of two major phyla, Bacteroidetes and Firmicutes, which account for more than $90 \%$ of the total population [27]. Since the relationship between the two major phyla, expressed as the B/F ratio, is related to several pathological conditions, this ratio has been used as an eventual biomarker [27]. Numerous studies have supported that higher F/B ratios are associated with a healthy state $[28,29]$, and lower B/F ratios are associated with obesity [30] and colorectal cancer [31,32]. Some studies have demonstrated that the gut microbiomes of obese animals and humans exhibit lower B/F ratios compared to normal-weight individuals $[28,31,32]$. In our previous study, Figure $1 B$, the $B /$ $F$ ratio lowered in 2-year-old male and female rats, suggesting the old age vulnerable to obesity and colorectal cancer compared to young age.

Sex differences influence not only the gut microbial composition but also the response to a change in host diet. In this study, the enterotypes in male and female rats were categorized differently. In the case of males, there were two enterotypes, E1 and E2. The male E1 included all normal diet groups regardless of their age and some 2-year-old HFDfed male rats, while the male E2 included the rest of the male rats in the HFD-fed groups. Similarly, in females, there were three enterotypes. The female E1 included almost all normal diet groups regardless of their age, and female E2 and E3 included the rest of the HFD-fed groups. 
A Male

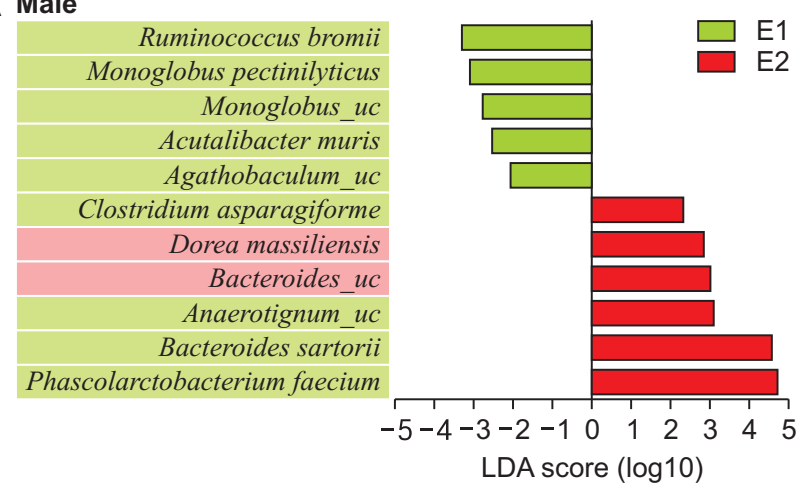

C Female

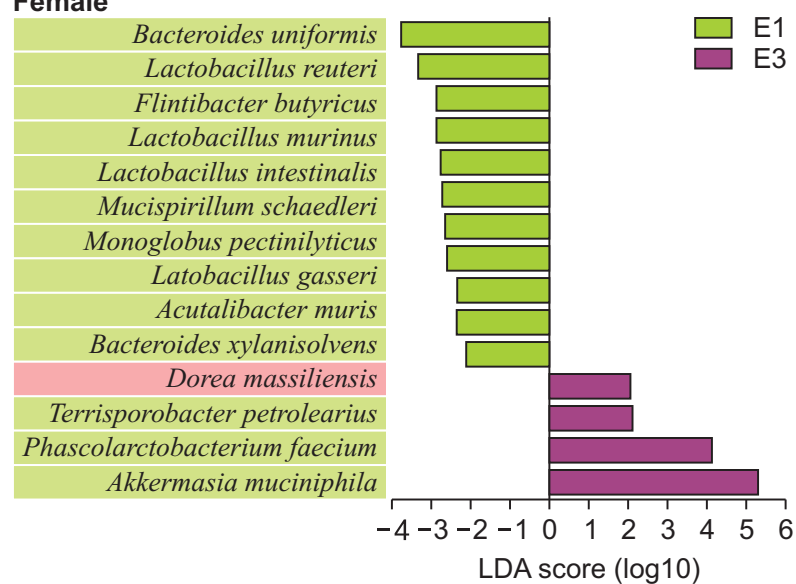

Commensal strains

Opportunistic pathogenic strains

\section{B Female}

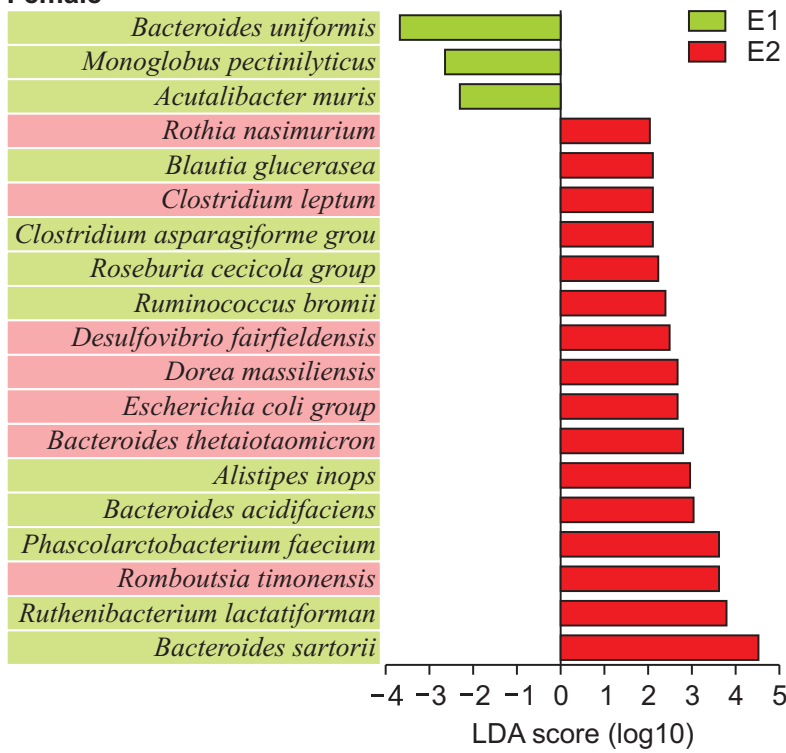

D Female

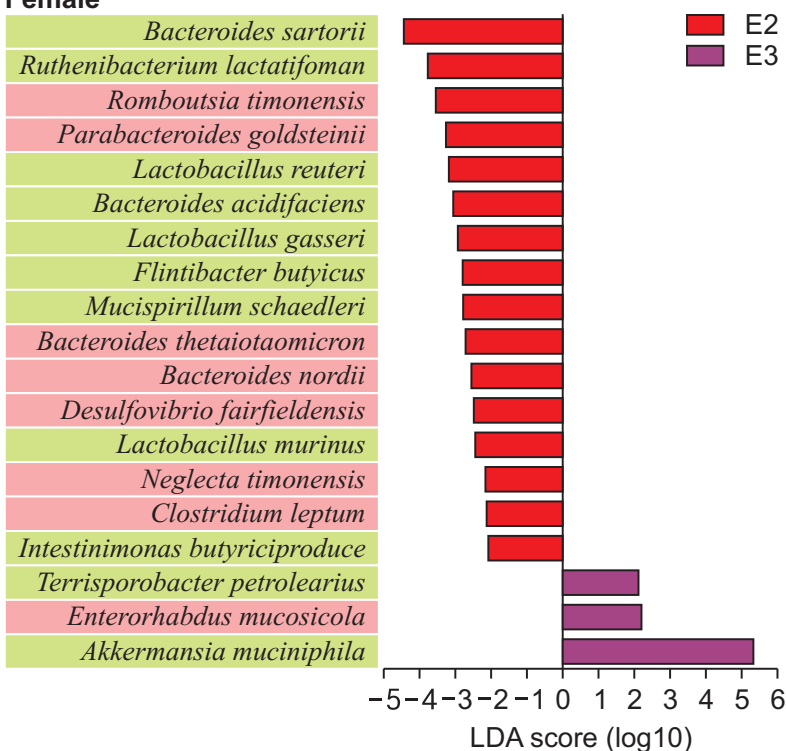

\begin{tabular}{ccccc}
\hline In panel & $\begin{array}{c}\text { Sample } \\
\text { information }\end{array}$ & $\begin{array}{c}\text { Commensal } \\
\text { strains }(\mathbf{n})\end{array}$ & $\begin{array}{c}\text { Opportunistic } \\
\text { pathogenic } \\
\text { strains (n) }\end{array}$ & $\begin{array}{c}\text { Opportunistic pathogenic } \\
\text { to commensal strains } \\
\text { (ratio) }\end{array}$ \\
\hline \multirow{2}{*}{$\mathbf{a}$} & Male E1 & 5 & 0 & $\mathbf{0}$ \\
& Male E2 & 4 & 2 & 0.5 \\
\hline \multirow{2}{*}{ b } & Female E1 & 3 & 0 & 0 \\
& Female E2 & 9 & 7 & 0.78 \\
\hline \multirow{2}{*}{$\mathbf{c}$} & Female E1 & 11 & 0 & 0 \\
& Female E3 & 3 & 1 & 0.33 \\
\hline \multirow{2}{*}{ d } & Female E2 & 9 & 7 & 0.78 \\
& Female E3 & 2 & 1 & 0.5 \\
\hline
\end{tabular}

Figure 5. The compositional alteration in the fecal microbiota of male and female rats. Bar plots of species with significant differences in abundance with linear effect size (LEfSe) analysis in male (A) and female (B-D) rats. Linear discriminant analysis (LDA) scores of species showed increased species in E1 (green), E2 (red), and E3 (purple) enterotypes. The ratio of opportunistic pathogenic to commensal strains was calculated and listed in (E). 

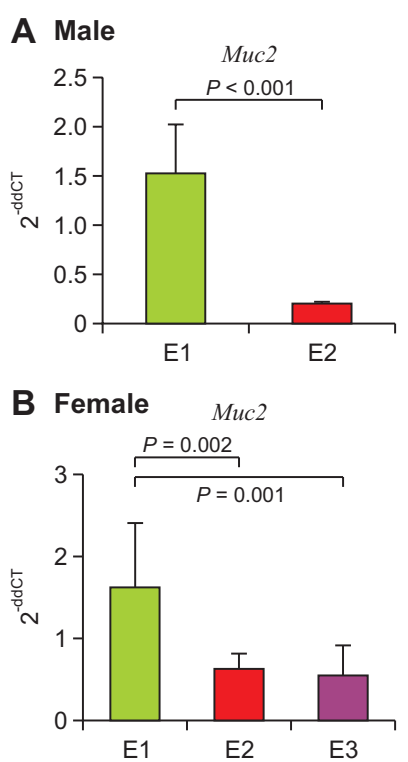
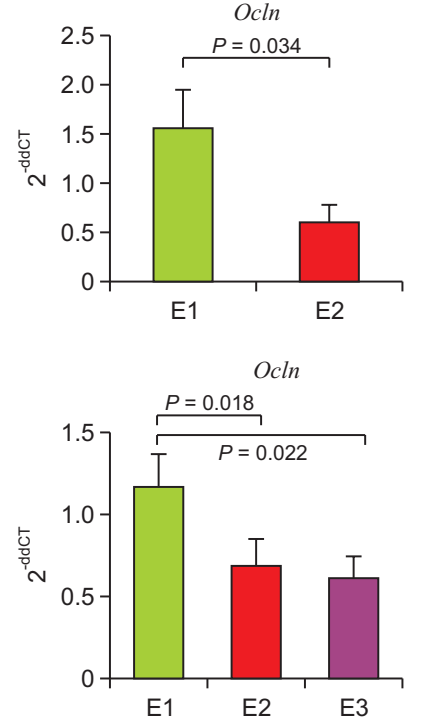
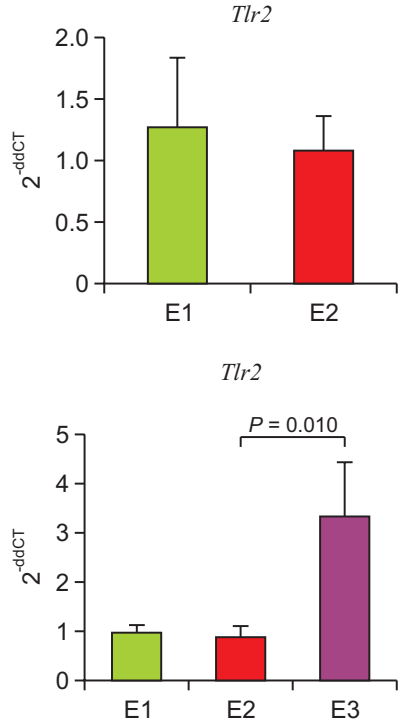

Figure 6. Differential host mRNA expression of Muc2, Ocln, and TIr2 in male and female enterotypes. The host colon mRNA expression of corresponding genes in the fecal microbiota of male $(A)$ and female (B) rats was measured by Realtime quantitative PCR (RT-qPCR) as described in MATERIALS AND METHODS. The statistical significance was calculated by the Mann-Whitney test. Muc2, Mucin 2; Ocln, Occludin; TIr2, Toll-like receptor 2 .
When we compared their relative abundance at the genus level, they were clearly classified into three types; male E1 and female E1 belong to the Prevotella type, male E2 and female E2 belong to the Bacteroides type, and female E3 belongs to the Ruminococcus type. These three taxa were reported as the dominant gut taxa contributing to the clustering of enterotypes in humans and represent the host's gut characteristics. For example, the fecal microbial community in humans who consume protein and animal fat for a long period is dominated by the Bacteroides genus, while Prevotella is dominant in long-term consumers of carbohydrates. In the case of the Ruminococcus genus, although it did not differentiate well from the Bacteroides and Prevotella genus, the Akkermansia muciniphila strain in their microbial community is well known. Similar to our data, male E2 and female E2 types, including most of the HFD-fed group, appear to be of the Bacteroides type in humans. In the case of the female E3 group, the high relative abundance of Akkermansia muciniphila may be close to the Ruminococcus type in humans $[22,33,34]$.

Animal microbiota studies had been criticized because of low relevance between animal and human microbiota [35]. Previously we reported that sex differences influence the response of the gut microbiome to an HFD, particularly in the 2-year-old age group but not in the 6-week-old F344 rats [17]. In the present study, we extended this analysis to the middle-aged group of 31- and 74- week-old rats in addition to the 6-week-old and the 2-year-old groups. In addition, we also performed an enterotype analysis, which uncovered a difference present in all age groups.

Moreover, when we analyzed mRNA expression of host colon depending on enterotypes, the female E3 group showed significantly higher mRNA expression of T/r2 compared to the female E2 group. Toll-like receptor 2 (TLR2) is a membrane receptor related to the immune system, recognizing pathogens, and activating innate immunity. It is known that intestinal epithelial TLR2 critically controls mucosal inflammation by directly preserving tight junction-associated barrier integrity [36], and the mechanism of barrier protective function is related to TLR2-mediated IL-10 production [16]. Moreover, the Ruminococcus genus, which is significantly high in the female E3 group, is well known to be categorized as a butyrate-producing bacteria [37] and influences IL-10 production [13]. In this context, we assume that the female E3 group represents the compensation ability of females to an HFD.

Akkermansia muciniphila is mucin-utilizing bacteria that colonize the mucin layer [38]. There was a report on the increase of Akkermansia in the cecal area of the HFD fed compared to the low-fat diet-fed rats [39]. Akkermansia muciniphila harbors all genes encoding enzymes involved in the citric acid cycle, which is important for fatty acid metabolism, while many bacteria species seem to lack genes for the whole cycle [40]. Therefore, we consider that the administration of fatty acid (HFD groups) contributes to colonization of Akkermansia muciniphila dominant in HFD groups.

This study has several limitations. The enterotype of normal mice with a homogeneous diet cannot reflect normal human gut microbiota due to fundamental differences in the diet pattern. It would be difficult to apply these results to human gut microbiota. However, it is nearly impossible to perform this kind of study regarding the effect of HFD on the gut microbiota in human beings. Therefore, we chose the rat model. As the aim of this study was to identify differences in the gut microbiome under a controlled diet in rats, we tried to cluster the HFD group only (Figure S1). However, we could not find consistent results. Thus, we compared the effect of HFD on gut microbiota by sex and age by enterotyping.

Also, there was no significant difference in the middle-age 
group of rats. From the results of our previous reports, only the older male group was influenced by an HFD, while younger males and females were unaffected [18]. Thus, we expected gradual microbial changes in the middle-age (31- and 74-week-old) group of rats. However, since it was impossible to follow every time point of aging, there was a huge time gap among different aged rats. However, we carried out this study in rats at 31 weeks of age and kept them under steady conditions with the corresponding diets until they became 74-week and 2-year-old. Therefore, we assume that the microbial changes may appear sharply in old age. Another limitation was the small number of 2-year-old male rats as the average life expectancy of a rat is nearly 2 years, and most rats naturally die by this time because of senescence $[41,42]$. In particular, older males tended to die earlier than females, and hence supplementing 2-year-old male rats was difficult. We assume that the lack of a compensation mechanism in older males, as observed in the female E3 enterotype, might account for the short life span of male rats.

Another limitation of this study is that the E3 enterotype appeared in some female rats even though the same HFD was given to the inbred rat strain. During the experiments, like the case of the male, we need to supply the enough number of 74-week and 2-year-old female rats to meet to analyze statistical significance properly. We consider that the old-aged female rats possess different microbiota initially because of the different batch, provoking manifestation of heterogenous enterotype, E3.

In the previous study, we found that aging itself affects the rodent's colon motility [42]. Another study has reported that HFD causes constipation in mice [43]. Moreover, some studies reported that constipation caused by HFD feeding is related to dysbiosis in colon mucus $[44,45]$. Therefore, the change of microbiota in our study may be associated with both aging and HFD. Furthermore, we could measure fecal consistency and amount to reveal the relation in constipation between aging and HFD.

In conclusion, HFD influenced fecal microbiota at all ages, regardless of sex, decreasing alpha and beta diversity. However, the shape of the fecal microbiota differed by sex in terms of enterotype clustering. In particular, female rats developed a compensatory enterotype response to an HFD.

\section{ACKNOWLEDGMENTS}

This work was supported by a grant from the National Research Foundation of Korea (NRF) funded by the government of the Republic of Korea (2019R1A2C2085149).

\section{CONFLICTS OF INTEREST}

No potential conflicts of interest were disclosed.

\section{SUPPLEMENTARY MATERIALS}

The raw unprocessed gene datasets of 16S rRNA, which were generated during the current study, are available with the NCBI Sequence Read Archive (SRA accession number: SRR 12052376 12052436), https://www.ncbi.nlm.nih.gov/ sra/PRJNA640490. The processed data generated and analyzed during this study has been included in this published article under Supplementary Dataset files. Supplementary materials can be found via https://doi.org/10.15430/ JCP.2021.26.4.277.

\section{ORCID}

Soo In Choi, https://orcid.org/0000-0001-6625-7608 Nayoung Kim, https://orcid.org/0000-0002-9397-0406 Ryoung Hee Nam, https://orcid.org/0000-0002-6515-4540 Ji Hyun Park, https://orcid.org/0000-0002-4809-8700 Heewon Nho, https://orcid.org/0000-0002-0324-0427 Jeong Eun Yu, https://orcid.org/0000-0003-4079-7360 Chin-Hee Song, https://orcid.org/0000-0002-3489-5944 Sun Min Lee, https://orcid.org/0000-0002-8438-3365 Dong Ho Lee, https://orcid.org/0000-0002-6376-410X

\section{REFERENCES}

1. Nagpal R, Mainali R, Ahmadi S, Wang S, Singh R, Kavanagh K, et al. Gut microbiome and aging: physiological and mechanistic insights. Nutr Healthy Aging 2018;4:267-85.

2. Amoroso C, Perillo F, Strati F, Fantini MC, Caprioli F, Facciotti F. The role of gut microbiota biomodulators on mucosal immunity and intestinal inflammation. Cells 2020;9:1234.

3. Leeming ER, Johnson AJ, Spector TD, Le Roy Cl. Effect of diet on the gut microbiota: rethinking intervention duration. Nutrients 2019;11:2862.

4. Claesson MJ, Jeffery IB, Conde S, Power SE, O'Connor EM, Cusack S, et al. Gut microbiota composition correlates with diet and health in the elderly. Nature 2012;488:178-84.

5. Labbé DP, Zadra G, Yang M, Reyes JM, Lin CY, Cacciatore S, et al. High-fat diet fuels prostate cancer progression by rewiring the metabolome and amplifying the MYC program. Nat Commun 2019;10:4358.

6. Shen W, Gaskins HR, Mclntosh MK. Influence of dietary fat on intestinal microbes, inflammation, barrier function and metabolic outcomes. J Nutr Biochem 2014;25:270-80.

7. Daniel H, Gholami AM, Berry D, Desmarchelier C, Hahne H, Loh $\mathrm{G}$, et al. High-fat diet alters gut microbiota physiology in mice. ISME J 2014;8:295-308.

8. Ding S, Chi MM, Scull BP, Rigby R, Schwerbrock NM, Magness $S$, et al. High-fat diet: bacteria interactions promote intestinal inflammation which precedes and correlates with obesity and insulin resistance in mouse. PLoS One 2010;5:e12191.

9. Guigoz Y, Doré J, Schiffrin EJ. The inflammatory status of old age can be nurtured from the intestinal environment. Curr Opin 
Clin Nutr Metab Care 2008;11:13-20.

10. Shin $\mathrm{JH}$, High KP, Warren CA. Older is not wiser, immunologically speaking: effect of aging on host response to clostridium difficile infections. J Gerontol A Biol Sci Med Sci 2016;71:916-22.

11. Rea MC, O'Sullivan O, Shanahan F, O'Toole PW, Stanton C, Ross RP, Hill C. Clostridium difficile carriage in elderly subjects and associated changes in the intestinal microbiota. J Clin Microbiol 2012;50:867-75.

12. Vlassoff C. Gender differences in determinants and consequences of health and illness. J Health Popul Nutr 2007;25:4761.

13. Kim YS, Unno T, Kim BY, Park MS. Sex differences in gut microbiota. World J Mens Health 2020;38:48-60.

14. Levast B, Li Z, Madrenas J. The role of IL-10 in microbiomeassociated immune modulation and disease tolerance. Cytokine 2015;75:291-301.

15. Shastri P, McCarville J, Kalmokoff M, Brooks SP, Green-Johnson JM. Sex differences in gut fermentation and immune parameters in rats fed an oligofructose-supplemented diet. Biol Sex Differ 2015;6:13.

16. Org E, Mehrabian M, Parks BW, Shipkova P, Liu X, Drake TA, et al. Sex differences and hormonal effects on gut microbiota composition in mice. Gut Microbes 2016;7:313-22.

17. Zheng L, Kelly CJ, Battista KD, Schaefer R, Lanis JM, Alexeev EE, et al. Microbial-derived butyrate promotes epithelial barrier function through IL-10 receptor-dependent repression of claudin-2. J Immunol 2017;199:2976-84.

18. Lee SM, Kim N, Yoon H, Nam RH, Lee DH. Microbial changes and host response in F344 rat colon depending on sex and age following a high-fat diet. Front Microbiol 2018;9:2236.

19. Choi SI, Son JH, Kim N, Kim YS, Nam RH, Park JH, et al. Changes in cecal microbiota and short-chain fatty acid during lifespan of the rat. J Neurogastroenterol Motil 2021;27:134-46.

20. Kwekel JC, Desai VG, Moland CL, Branham WS, Fuscoe JC. Age and sex dependent changes in liver gene expression during the life cycle of the rat. BMC Genomics 2010;11:675.

21. Quinn R. Comparing rat's to human's age: how old is my rat in people years? Nutrition 2005;21:775-7.

22. Arumugam M, Raes J, Pelletier E, Le Paslier D, Yamada T, Mende DR, et al. Enterotypes of the human gut microbiome. Nature 2011;473:174-80.

23. Liu Y, Li Z, Xiong H, Gao X, Wu J. Understanding of internal clustering validation measures. Presented at the 10th IEEE International Conference on Data Mining, ICDM 2010, Sydney, Australia, December 14-17, 2010. p.911-6.

24. Segata N, Izard J, Waldron L, Gevers D, Miropolsky L, Garrett WS, et al. Metagenomic biomarker discovery and explanation. Genome Biol 2011;12:R60.

25. Choi SI, Kim N, Lee SM, Nam RH, Kang SR, Song CH, et al. Rat intestinal acetic acid and butyric acid and effects of age, sex, and high-fat diet on the intestinal levels in rats. J Cancer Prev 2019;24:20-5.

26. Lathrop SK, Bloom SM, Rao SM, Nutsch K, Lio CW, Santacruz $\mathrm{N}$, et al. Peripheral education of the immune system by colonic commensal microbiota. Nature 2011;478:250-4.

27. Magne F, Gotteland M, Gauthier L, Zazueta A, Pesoa S, Navarrete $P$, et al. The Firmicutes/Bacteroidetes ratio: a relevant marker of gut dysbiosis in obese patients? Nutrients 2020;12: 1474.

28. Bervoets L, Van Hoorenbeeck K, Kortleven I, Van Noten C, Hens N, Vael C, et al. Differences in gut microbiota composition between obese and lean children: a cross-sectional study. Gut Pathog 2013;5:10.

29. Ley RE, Turnbaugh PJ, Klein S, Gordon JI. Microbial ecology: human gut microbes associated with obesity. Nature 2006; 444:1022-3.

30. Raskov H, Burcharth J, Pommergaard HC. Linking gut microbiota to colorectal cancer. J Cancer 2017;8:3378-95.

31. Ley RE, Bäckhed F, Turnbaugh P, Lozupone CA, Knight RD, Gordon Jl. Obesity alters gut microbial ecology. Proc Natl Acad Sci USA 2005;102:11070-5.

32. Turnbaugh PJ, Hamady M, Yatsunenko T, Cantarel BL, Duncan $A$, Ley RE, et al. A core gut microbiome in obese and lean twins. Nature 2009;457:480-4.

33. Costea PI, Hildebrand F, Arumugam M, Bäckhed F, Blaser MJ, Bushman FD, et al. Enterotypes in the landscape of gut microbial community composition. Nat Microbiol 2018;3:8-16.

34. Wu GD, Chen J, Hoffmann C, Bittinger K, Chen YY, Keilbaugh $\mathrm{SA}$, et al. Linking long-term dietary patterns with gut microbial enterotypes. Science 2011;334:105-8.

35. Nguyen TL, Vieira-Silva S, Liston A, Raes J. How informative is the mouse for human gut microbiota research? Dis Model Mech 2015;8:1-16.

36. Cario E. Barrier-protective function of intestinal epithelial Toll-like receptor 2. Mucosal Immunol 2008;1 Suppl 1:S62-6.

37. Takahashi K, Nishida A, Fujimoto T, Fujii M, Shioya M, Imaeda $\mathrm{H}$, et al. Reduced abundance of butyrate-producing bacteria species in the fecal microbial community in Crohn's disease. Digestion 2016;93:59-65.

38. Derrien M, Vaughan EE, Plugge CM, de Vos WM. Akkermansia muciniphila gen. nov., sp. nov., a human intestinal mucindegrading bacterium. Int J Syst Evol Microbiol 2004;54(Pt 5): 1469-76.

39. Zhong $Y$, Marungruang N, Fåk F, Nyman M. Effects of two whole-grain barley varieties on caecal SCFA, gut microbiota and plasma inflammatory markers in rats consuming low- and highfat diets. Br J Nutr 2015;113:1558-70.

40. Kanehisa M, Goto S. KEGG: kyoto encyclopedia of genes and genomes. Nucleic Acids Res 2000;28:27-30.

41. Kang JM, Kim N, Kim JH, Oh E, Lee BY, Lee BH, et al. Effect of aging on gastric mucosal defense mechanisms: ROS, apoptosis, angiogenesis, and sensory neurons. Am J Physiol Gastrointest Liver Physiol 2010;299:G1147-53.

42. Lee SM, Kim N, Jo HJ, Park JH, Nam RH, Lee HS, et al. Comparison of changes in the interstitial cells of Cajal and neuronal nitric oxide synthase-positive neuronal cells with aging between the ascending and descending colon of F344 rats. J Neurogastroenterol Motil 2017;23:592-605. 
43. Mukai R, Handa O, Naito Y, Takayama S, Suyama Y, Ushiroda $C$, et al. High-fat diet causes constipation in mice via decreasing colonic mucus. Dig Dis Sci 2020;65:2246-53.

44. Hwang N, Eom T, Gupta SK, Jeong SY, Jeong DY, Kim YS, et al. Genes and gut bacteria involved in luminal butyrate reduction caused by diet and loperamide. Genes (Basel) 2017;8:350.

45. Anitha M, Reichardt F, Tabatabavakili S, Nezami BG, Chassaing $B$, Mwangi S, et al. Intestinal dysbiosis contributes to the delayed gastrointestinal transit in high-fat diet fed mice. Cell Mol Gastroenterol Hepatol 2016;2:328-39. 